\title{
Fuzzy Based Adaptive User-Weight Classification Scheme for EDCA in IEEE 802.11e WLAN
}

\author{
${ }^{*}$ V. Ilayaraja ${ }^{1}$ and R. Venkatesan ${ }^{2}$ \\ ${ }^{1}$ Assistant Professor (Sr.Gr), Department of Information Technology, \\ PSG College of Technology, Coimbatore, Tamilnadu, India. \\ ${ }^{2}$ Professor and Head, Department of Computer Science and Engineering, \\ PSG College of Technology, Coimbatore, Tamilnadu, India. \\ Iinfoilai11@gmail.com
}

\begin{abstract}
In IEEE 802.11e Wireless Local Area Network (WLAN), the existing userdifferentiation technique used in Enhanced Distributed Channel Access (EDCA) protocol is affected by the collision rate and battery capacity of the mobile stations. To solve this issue, a Fuzzy based user-weight classification scheme for EDCA in IEEE 802.11e WLAN is proposed. In this scheme, the inter-node priority for each traffic class is assigned to each mobile station. To estimate the inter-node priority, fuzzy logic is applied considering the traffic access category, number of transmitted packets, collision rate and residual energy level parameters for each traffic class. Based on the outcome of the fuzzy rules, inter-node priority is determined and the node weights are updated dynamically. From simulation results, it is seen that the proposed technique reduces waiting time and maximizes the throughput when compared with the QoS Maximization of EDCA (QMEDCA) technique.
\end{abstract}

Keywords: IEEE 802.11e, Wireless Local Area Network (WLAN), Quality of Service (QoS), Enhanced Distributed Channel Access (EDCA), Fuzzy Logic, Priority, Traffic Access Category

\section{Introduction}

In Wireless LAN, IEEE 802.11e standard is used for time-sensitive applications to offer Quality of Service (QoS) support. It specifies a Hybrid Coordination Function (HCF), which is a new coordination function under the control of a Hybrid Coordinator (HC). HC positioned in the QoS Access Point (QAP) has two channel access modes: Enhanced Distributed Channel Access (EDCA) and HCF-Controlled Channel Access (HCCA). EDCA depends on a distributed control thus enabling prioritized channel access [1]. Stations operating under 802.11e specifications are usually known as QoS Stations (QSTAs) [2-3].

IEEE 802.11e standard is an improvised version of the fundamental IEEE 802.11 MAC which is being standardized. QoS is ensured in IEEE 802.11e by employing the priority scheme. When compared with the original version, IEEE 802.11e does not consider all the data packets with same priority. In IEEE 802.11e, on the basis of the QoS needs, the data packets are prioritized. For every priority of data, there are four Access Categories (AC). On the basis of the data priority, medium access is granted such that data with specific priority is linked to a specific AC. The contention for the medium is based on some contention factors of each $\mathrm{AC}$ and service differentiation is provided accordingly [4].

In the EDCA method, some MAC layer parameters which are used to provide priority level to each AC are the Arbitration Inter-Frame Space (AIFS), Transmission Opportunity 
(TXOP) and Contention Window minimum (CWmin) and Contention Window maximum (CWmax) [5].

\subsection{Problem Identification}

EDCA of the IEEE 802.11e standard is beneficial for prioritizing the traffic. In EDCA, packet access qualifications are considered only for intra-node traffic streams and not provided for different user stations in the network. In other words, no distinction has been taken into account by the standard mechanism between two active stations that are transmitting data packets simultaneously within same Access Category (AC).

Several existing works have utilized fuzzy logic as the basis of their proposed technique, because of its ability to imitate human decisions and also for its simplicity of use and implementation. Since the inter-node priority depends on various conflicting parameters, using fuzzy logic for decision model will help to arrive a clear solution.

In [6], QoS Maximization of EDCA (QM-EDCA) which is an enhanced version of EDCA is developed based on fuzzy logic. QM-EDCA dynamically adapts the Arbitration inter frame Spaces (AIFS) according to the network state and remaining energy. It has three inputs to the fuzzy logic theory, which are Collision Counter (CC), Sent Packet Counter (SPC) and Remaining Energy of Battery (REB). Since service classes are not strictly enforced, sometimes the servicing of low priority traffic can degrade the service designed to high priority traffic. But, Service Differentiation should be done depending on the real network conditions by classifying the stations rather than the traffic. No flexible QoS mechanism exists that could dynamically adjust the QoS specification based on network conditions [7].

Also, in IEEE 802.11e WLAN, the existing user-differentiation technique in EDCA protocol [8] is affected by the collision rate and battery capacity of the different stations.

To overcome the above highlighted issues, a Fuzzy based user-weight classification scheme for EDCA in IEEE 802.11e WLAN is proposed.

\section{Related Works}

Pingping Dong et al [9] have presented a novel Medium Access Control (MAC) scheme using Balanced EDCA (BEDCA). It provides service differentiation between the access point (AP) and the mobile stations (STAs) to enhance the Voice over Internet Protocol (VoIP) capacity. In BEDCA, an expression for the contention window of AP is determined which is independent of the participating STAs. It differentiates the service between the AP and STAs by allocating a higher priority Access Category (AC) for the AP so that it attains higher channel access probability compared to a STA.

Sunmyeng Kim [10] proposed a novel Cooperative MAC protocol for QoS enhancement (QC-MAC) in WLANs based on EDCA. In this protocol, a node transmits multiple data packets consecutively until the transmission period becomes greater than the specific TXOP time period. Receiver acknowledges a block of received data packets using the Block Acknowledgement (ACK) procedure.

Pablo Serrano et al [11] have developed an algorithm to compute the optimal configuration of the EDCA parameters based on the throughput and delay requirements of the stations. It takes the input traffic requirements from both real time and non real-time stations and outputs the configuration that maximizes the performance.

Kang Yong Lee et al [12] have proposed Explicit Traffic Aware scheduling algorithm with Explicit Queue Length Notification (ETA-EQN) frame for supporting multimedia services in HCCA of IEEE 802.11e WLAN. Each station sends a special frame called EQN to the QAP before the end of every SI which consists of the queue length information of its own. During the starting of the SI, the QAP uses the queue length information that consisting of most recent queue length in EQN frames to calculate TXOPs of each station through EDCA function. To reduce the waste of the HCCA time 
resource, the EQN frame is sent using EDCA channel access scheme with the first priority and is queued at the head of the queue to ensure immediate transmission. Then the QAP gathers the queue length information of each station by receiving and retrieving information from the EQN frames.

Saeid Montazeri et al [13] have proposed Adaptive Fair Distributed Scheduling algorithm (AFDSA) for IEEE 802.11e. This algorithm extended the RTS/CTS frame by including three new parts to ensure QoS. When a specific station reserves a channel at a given time interval, the remaining stations contend for gaining channel access. This algorithm ensures QoS for both VBR and CBR traffic.

Fakhar Uddin Ahmed and Shikhar Kr. Sarma [14] have developed QAP based admission control technique which is used to guarantee proper use of the network resources and QoS. This mechanism overcomes the load instability issue between the QAP involved with BSS. The inter QAP communication process within bigger WLAN is deployed along with the admission control unit in EDCA. QoS management techniques developed on the basis of the inter QAP differentiation causes proper use of the resources by relocating the QSTA from highly loaded region to minimally loaded QAP. This assures QoS for different kinds of traffic.

Feyza Keceli et al [15] have designed Weighted Fair Uplink/Downlink Access Provisioning mechanism which is basically an EDCA parameter adaptation algorithm. This mechanism maintains the priority between the AC and also attains the predefined utilization ratio among the uplink and downlink traffic flow. The major advantage of this mechanism is that it is a unique mechanism as the EDCA factors are robustly adjusted based on the estimation depending on the active link count as well as the utilization ratio.

\section{Proposed Solution}

\subsection{Overview}

In this paper, a Fuzzy based user-weight classification scheme for EDCA in IEEE 802.11e WLAN is proposed. In this scheme, the inter-node priority for each traffic class is assigned to each mobile station. Fuzzy logic is applied considering the traffic access category, number of transmitted packets, collision rate and residual energy level parameters for each traffic class to estimate inter-node priority. Based on the outcome of the Fuzzy rules, inter node priority is determined and the node weights are updated dynamically.

\subsection{Estimation of Metrics}

The important metrics, traffic Access Category (AC), Collision Rate $\left(\mathrm{R}_{\mathrm{c}}\right)$, and Residual Energy level $\left(\mathrm{E}_{\mathrm{res}}\right)$ are presented in this section.

3.2.1. Traffic Access Category (AC): EDCA uses four access categories which are given below. These categories are queues of Drop-Tail type which uses the First In First Out (FIFO) technique. Each queue contains channel access priority level.

- $\quad$ Voice - High Priority

- $\quad$ Video - Medium Priority

- $\quad$ Best Effort - Medium Priority

- $\quad$ Background - Low Priority

3.2.2. Collision Rate: The collision rate is computed using the following equation:

$$
\mathrm{R}_{\mathrm{c}}=\frac{N_{c}(t)}{N_{t x}(t)} \times 100 \%
$$


where, $\mathrm{N}_{\mathrm{c}}$ denotes the number of collisions and $\mathrm{N}_{\mathrm{tx}}$ denotes the number of packets sent at time $\mathrm{t}$.

3.2.3. Residual Energy Level: The residual energy level is estimated using the following equation:

$$
\mathrm{E}_{\mathrm{res}}=\frac{R E(t)}{T E(t)} \times 100 \%
$$

where, RE is the remaining battery energy and TE is the total battery energy at time $t$.

\subsection{Fuzzy Based Adaptive User-weight Classification Scheme}

In this scheme, the inter-node priority for each traffic class is assigned to each mobile station. In order to estimate the inter-node priority, the fuzzy logic is applied considering the following input parameters: (estimated as in sections 3.2.2 \& 3.2.3)

- Traffic Access Category (AC)

- Number of Transmitted Packets $\left(\mathrm{N}_{\mathrm{tx}}\right)$

- Collision Rate $\left(\mathrm{R}_{\mathrm{c}}\right)$

- Residual Energy Level $\left(\mathrm{E}_{\mathrm{res}}\right)$

Based on the outcome of the Fuzzy rules, inter node priority is determined and the node weights are updated dynamically.

The steps involved in the fuzzy logic decision making are: Fuzzification, Rule Evaluation, Rule Aggregation and Defuzzification. The fuzzy logic decision system is shown in Figure 1.

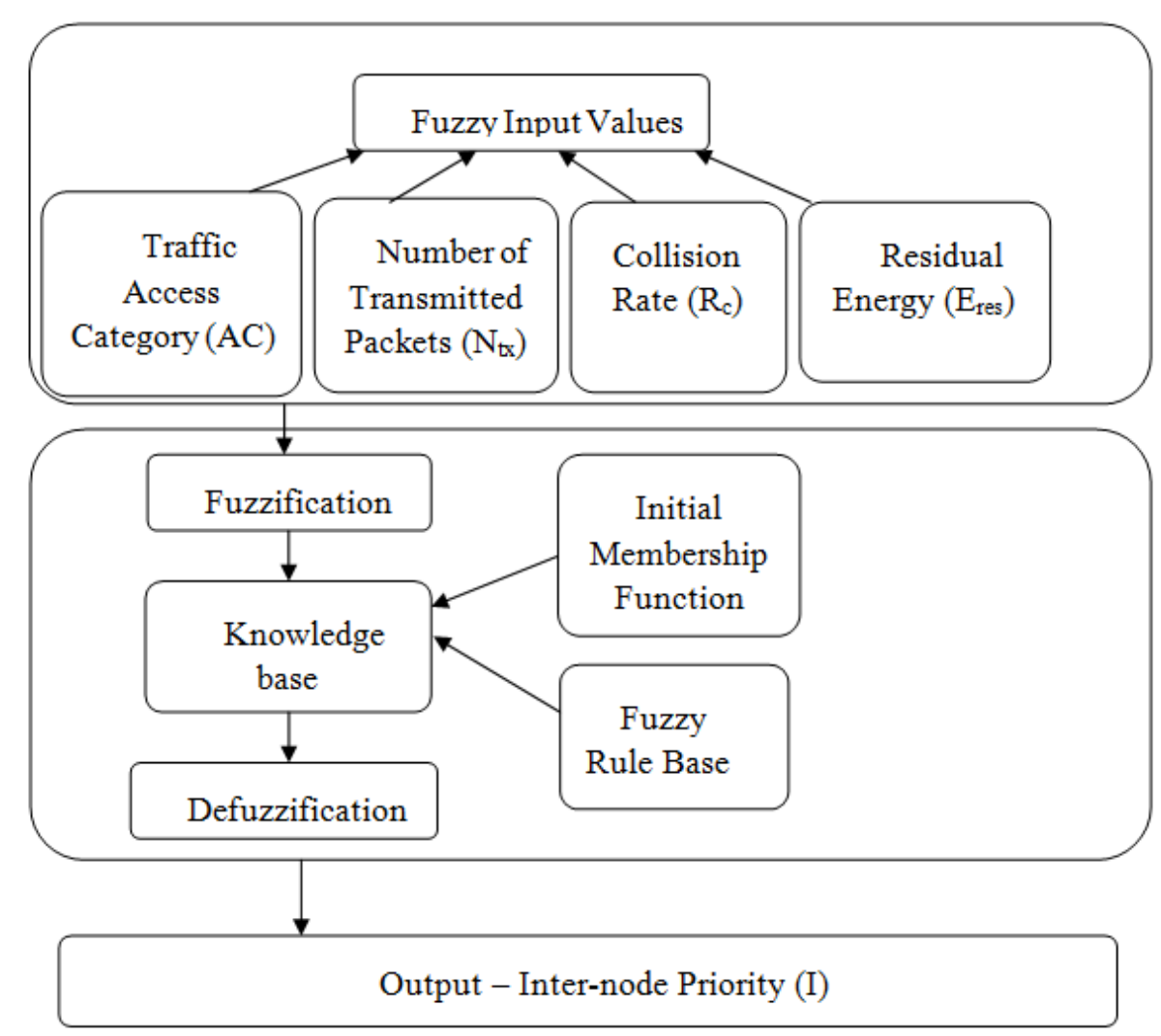

Figure 1. Fuzzy Logic Decision System 


\section{Fuzzification}

The input variables Traffic Access Category (AC), Number of Transmitted Packets $\left(\mathrm{N}_{\mathrm{tx}}\right)$, Collision Rate $\left(\mathrm{R}_{\mathrm{c}}\right)$ and Residual Energy Level $\left(\mathrm{E}_{\mathrm{res}}\right)$ are given a degree to appropriate fuzzy sets. The linguistic values, high, medium and low for each of $\mathrm{AC}, \mathrm{N}_{\mathrm{tx}}$, $\mathrm{R}_{\mathrm{c}}$ and $\mathrm{E}_{\mathrm{res}}$ are taken.

Figures 2 to 6 show the fuzzy sets along with graph of membership functions for the input and output variables. Since three linguistic values are considered, the triangular fuzzy set is applied for each membership function.

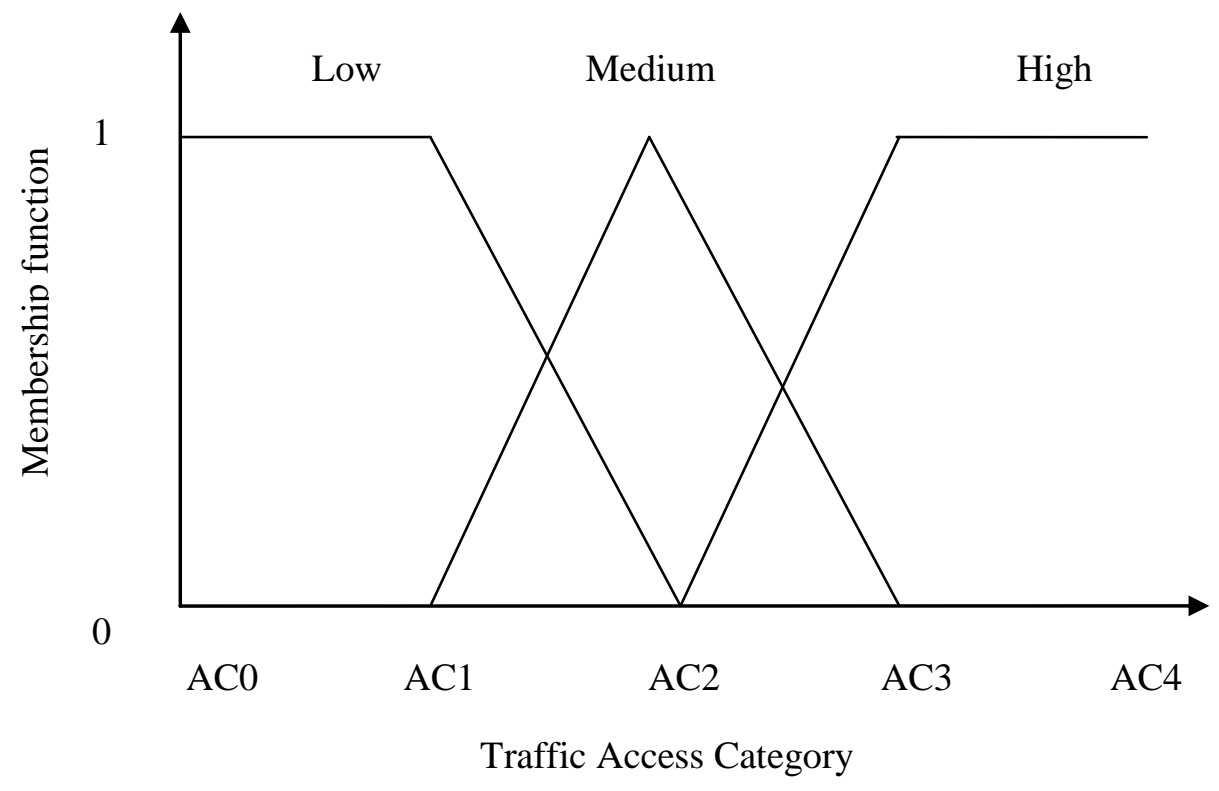

Figure 2. Membership Function of Traffic Access Category

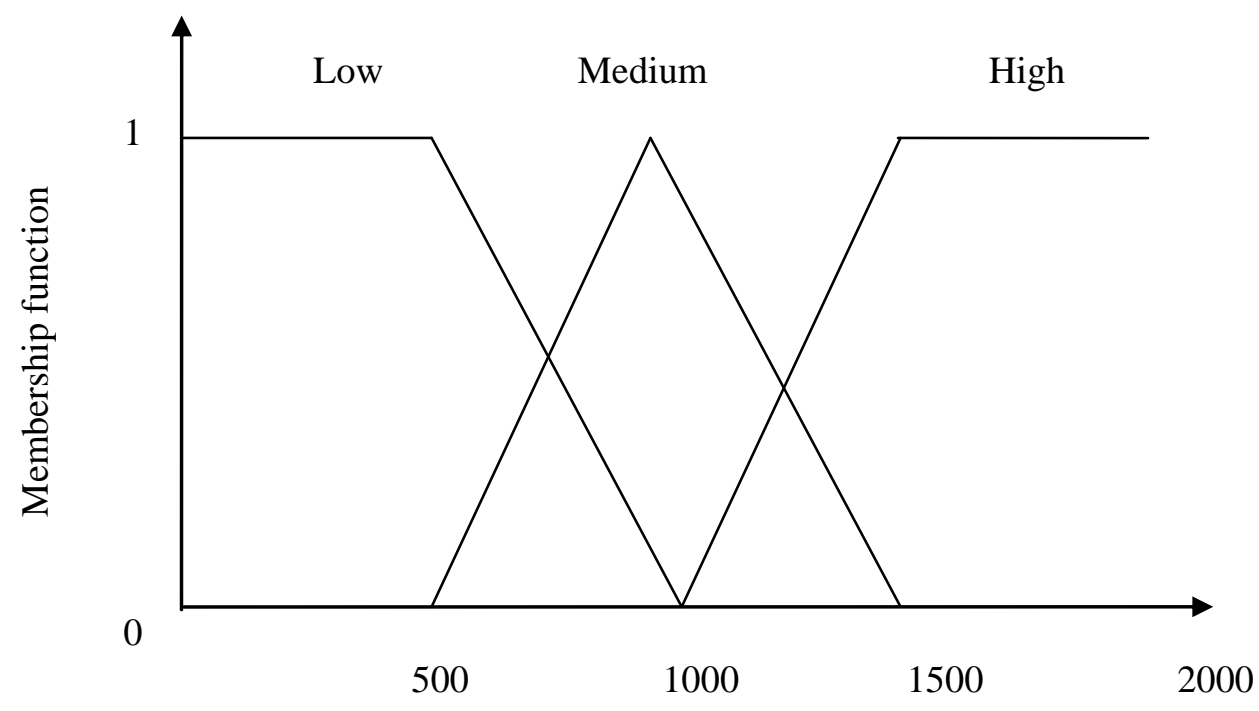

Number of Transmitted Packets

Figure 3. Membership Function of Transmitted Packet Count 


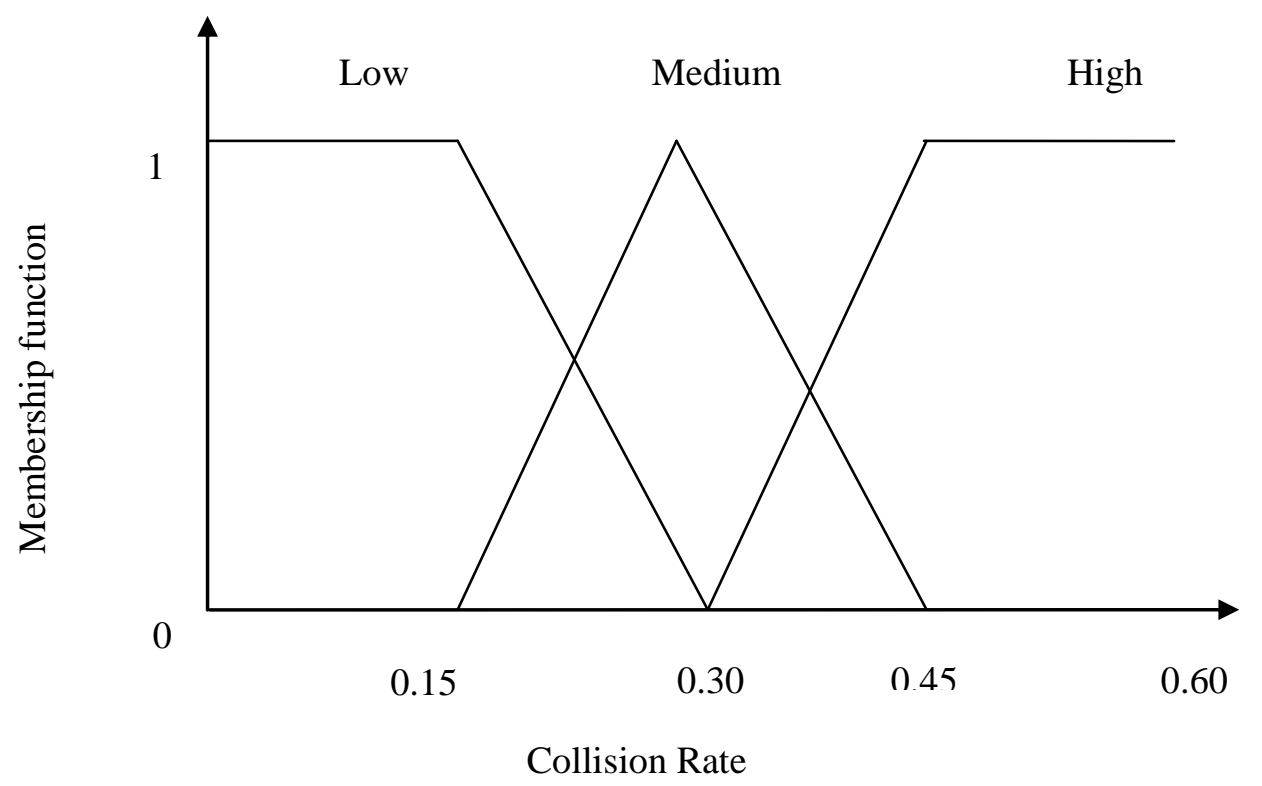

Figure 4. Membership Function of Collision Rate

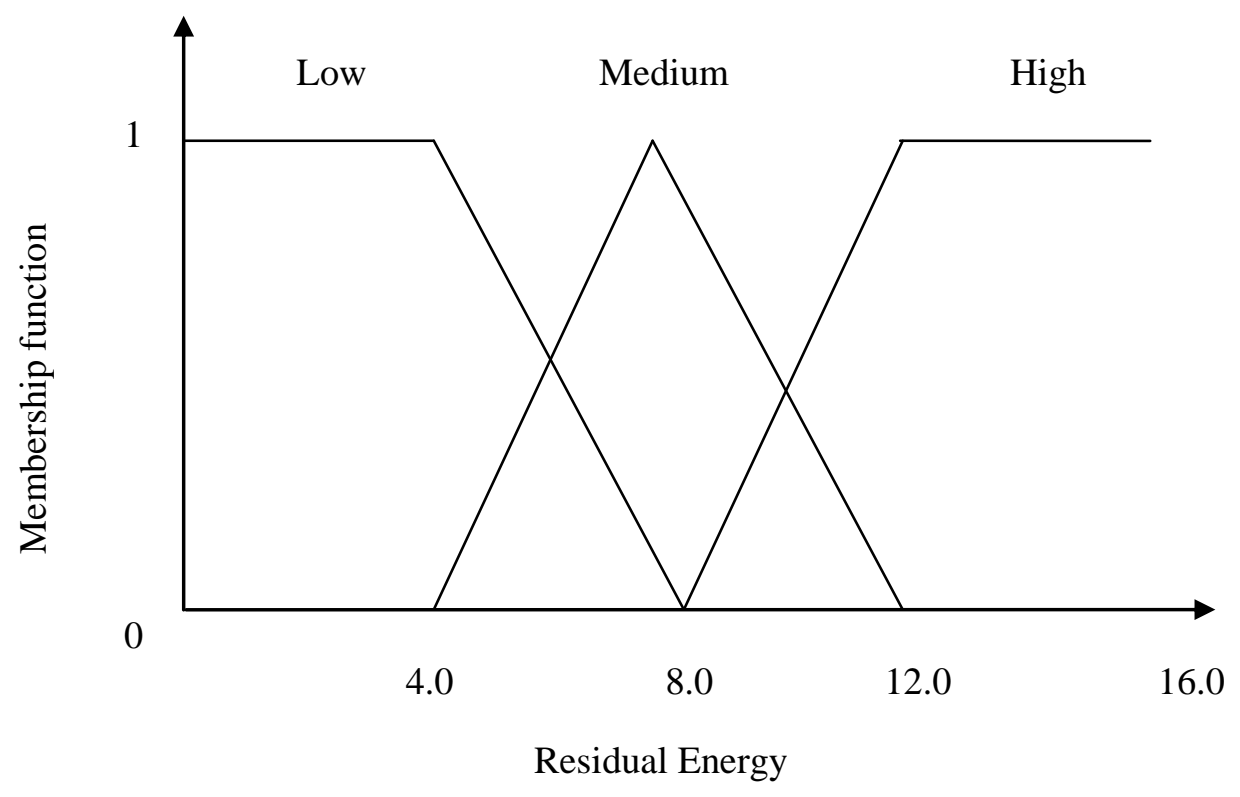

Figure 5. Membership Function of Residual Energy 


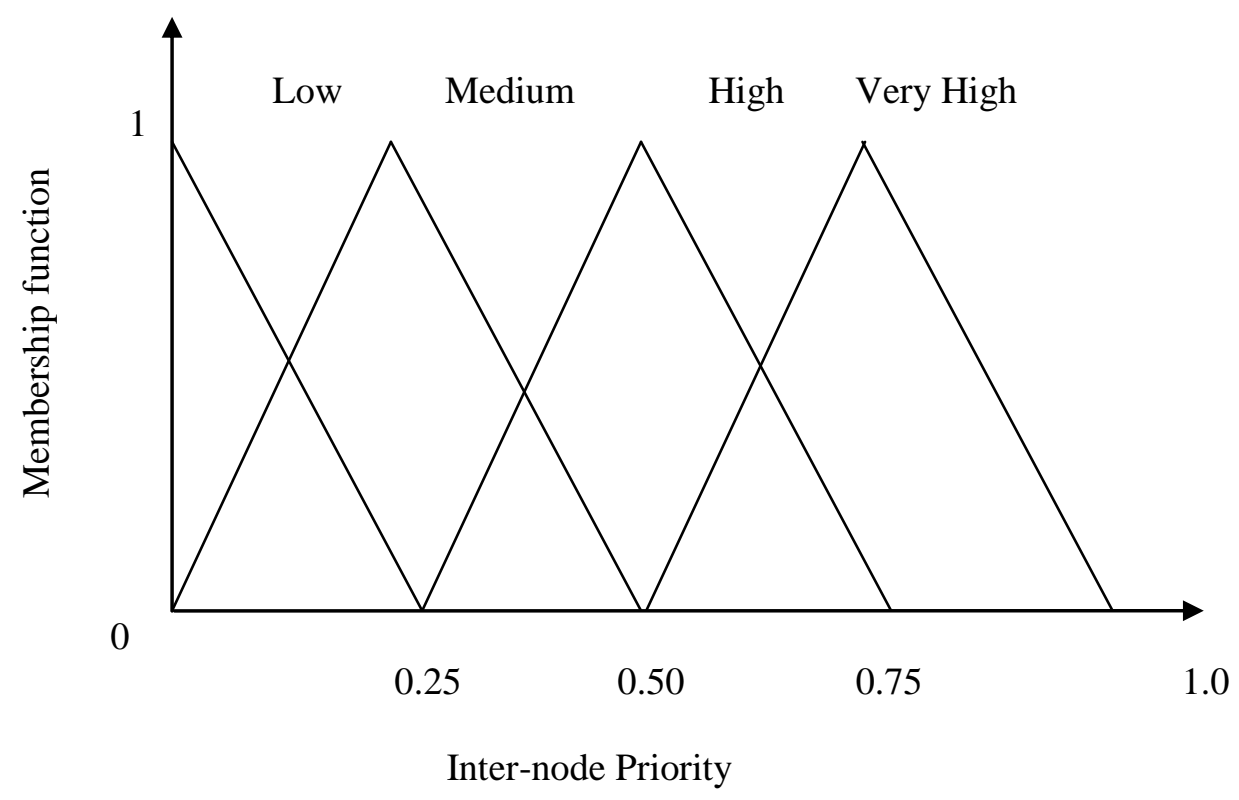

Figure 6. Membership Function of Inter-Node Priority Level

As seen in table 1, if $A C, N_{t x}, R_{c}$ and $E_{\text {res }}$ are the inputs, then the output represents the inter-node priority. The fuzzy rules are defined as given in table 1. The output of each rule is combined to generate the fuzzy decision.

Table 1. Fuzzy Rule Base

\begin{tabular}{|c|c|c|c|c|c|}
\hline S.No & $\begin{array}{c}\text { Traffic } \\
\text { Access } \\
\text { Category } \\
\text { (AC) }\end{array}$ & $\begin{array}{c}\text { Transmitted } \\
\text { Packet Count } \\
\left(\mathbf{N}_{\mathrm{tx}}\right)\end{array}$ & $\begin{array}{l}\text { Collision } \\
\text { Rate }\left(\mathbf{R}_{\mathrm{c}}\right)\end{array}$ & $\begin{array}{c}\text { Residual } \\
\text { Energy } \\
\left(\mathbf{E}_{\mathrm{res}}\right)\end{array}$ & $\begin{array}{l}\text { Inter-node } \\
\text { Priority (I) }\end{array}$ \\
\hline 1 & Low & Low & Low & High & Low \\
\hline 2 & Low & Low & High & High & Medium \\
\hline 3 & Low & High & High & Low & High \\
\hline 4 & Low & High & Low & High & Low \\
\hline 5 & Low & High & High & High & Medium \\
\hline 6 & High & High & High & Low & Very High \\
\hline 7 & High & High & Low & High & Medium \\
\hline 8 & High & High & High & High & High \\
\hline 9 & High & Low & Low & High & Low \\
\hline 10 & High & Low & Low & Low & Medium \\
\hline 11 & High & Low & High & Low & High \\
\hline 12 & High & Low & High & High & Medium \\
\hline 13 & Medium & High & High & Low & High \\
\hline 14 & Medium & High & Low & High & Low \\
\hline 15 & Medium & High & Low & Low & Medium \\
\hline 16 & Medium & Low & Low & High & Low \\
\hline 17 & Medium & Low & Low & Low & Low \\
\hline 18 & Medium & Low & High & High & Low \\
\hline 19 & Medium & Low & High & Low & Medium \\
\hline 20 & Medium & High & High & High & Medium \\
\hline
\end{tabular}


The basic of fuzzy rule formation can be explained as follows. The nodes with high access category of traffic having high transmitted packet count, high collision rate and low residual energy indicates that the nodes will soon drain out of energy due to overload of queue and collision. Hence those nodes transmitting higher access category of traffic should be granted the highest inter-node priority. On the other hand, if the traffic access category is low with high residual energy, less packets transmission and low collision, then it can be scheduled later, granting lowest inter-node priority. Based on these criteria, table 1 contains 20 rules.

For example,

According to Rule 6,

If $\left(\mathrm{AC}, \mathrm{N}_{\mathrm{tx}}, \& \mathrm{R}_{\mathrm{c}}=\right.$ High $) \&\left(\mathrm{E}_{\mathrm{res}}=\right.$ Low $)$, Then

End if Inter-node priority is very high.

\section{Defuzzification:}

The technique by which a crisp value is extracted from the output fuzzy set is defined as defuzzification. The centroid of area scheme is applied for defuzzification during fuzzy decision making process. The Equation (3) describes the defuzzifier method.

$$
\text { Fuzzy_cost }=\left[\sum_{\text {allrules }} f_{i} * \alpha\left(\mathrm{f}_{\mathrm{i}}\right)\right] /\left[\sum_{\text {allrules }} \alpha\left(f_{i}\right)\right]
$$

where, fuzzy_cost is used to specify the degree of decision making, $\mathrm{f}_{\mathrm{i}}$ is the sum of all fuzzy rules, and $\alpha\left(f_{i}\right)$ is its membership function.

Based on the inter-node priority level, the node weight is dynamically adjusted. This in turn minimizes the waiting delay and maximizes the aggregate throughput.

\section{Simulation Results}

\subsection{Simulation Parameters}

The proposed Fuzzy based Adaptive User-weight Classification Scheme for EDCA (FAUCS) is simulated in NS-2. Table 2 shows the simulation settings and parameters used.

Table 2. Simulation Parameters

\begin{tabular}{|c|c|}
\hline Parameter & Value \\
\hline Number of QoS Stations & $6,8,10$ and 12 \\
\hline Topology Size & $100 \mathrm{~m} \mathrm{X} \mathrm{100m}$ \\
\hline Simulation Time & $60 \mathrm{sec}$ \\
\hline Propagation & TwoRayGround \\
\hline Antenna & OmniAntenna \\
\hline Initial Energy & 15 Joules \\
\hline Transmission Power & 0.3 watts \\
\hline Receiving Power & 0.5 watts \\
\hline Traffic Source & CBR and VBR \\
\hline Transmission Rate & $250 \mathrm{~kb}, 500 \mathrm{~kb}, 750 \mathrm{~kb}$, \\
$1000 \mathrm{~kb}, 1250 \mathrm{~kb}$ and $1500 \mathrm{~Kb}$ \\
\hline
\end{tabular}

\subsection{Performance Metrics}

The performance of the proposed FAUCS protocol is evaluated on the basis of the following parameters and compared with the QoS Maximization of EDCA (QM-EDCA) [6] protocol.

- Delay (sec): It is total time taken by the packets to reach the destination. 
- Packet Drop : It is the number of packets dropped during the data transmission.

- Bandwidth Allocation (Mb/s): It is the amount of bandwidth allocated by QAP to each QSTA.

- Bandwidth Utilization $(\mathbf{M b} / \mathbf{s})$ : It is the amount of bandwidth utilized by each QSTA.

- $\quad$ Residual Energy (Joules): It is the amount of energy that remains in the nodes after data transmission.

\subsection{Results \& Analysis}

4.3.1. Based on QoS Stations: In the first experiment, the number of users is varied as 6 , 8,10 and 12 .

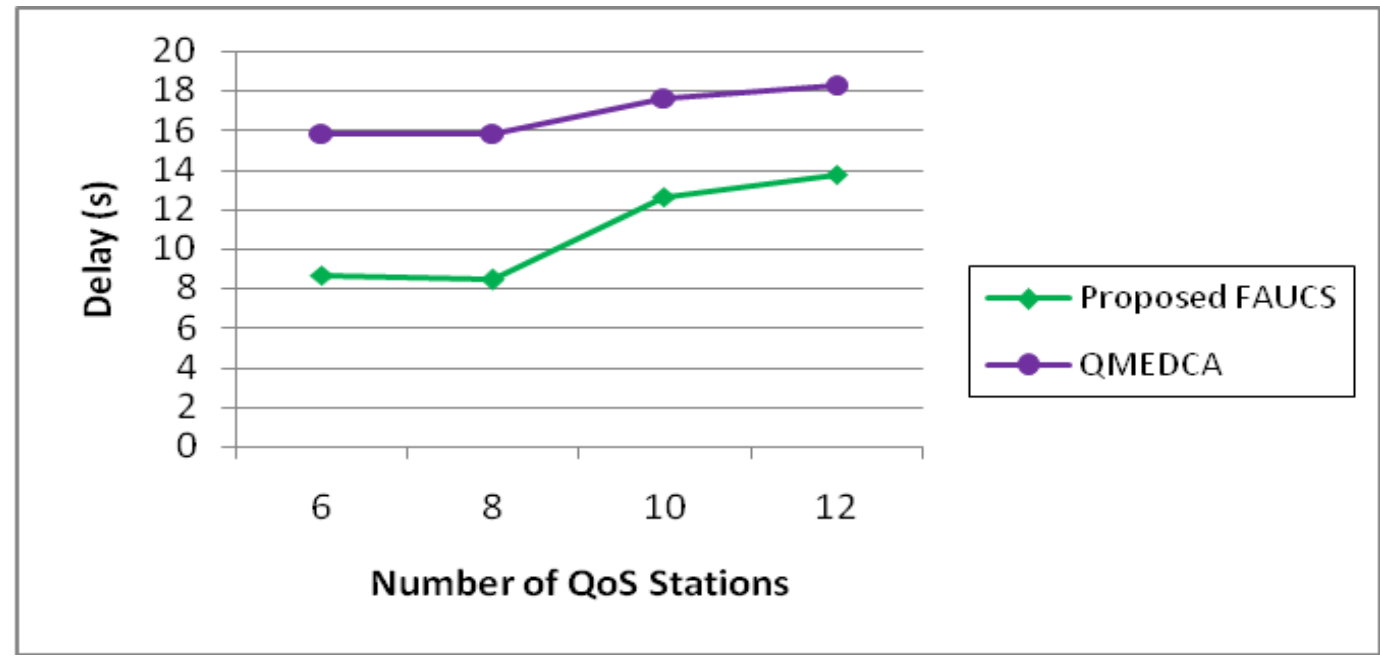

Figure 7. QoS Stations Vs Delay

The graph in Figure 7 shows the results of delay occurred for FAUCS and QMEDCA techniques when the number of QSTAs is varied. When increasing QSTAs, delay is also increases. As seen from the figure, the delay of FAUCS is increased from $8.65 \mathrm{sec}$ to $13.77 \mathrm{sec}$ and the delay of QMEDCA is increases from $15.81 \mathrm{sec}$ to $18.28 \mathrm{sec}$. Hence, FAUCS has 36\% lesser delay than QMEDCA technique.

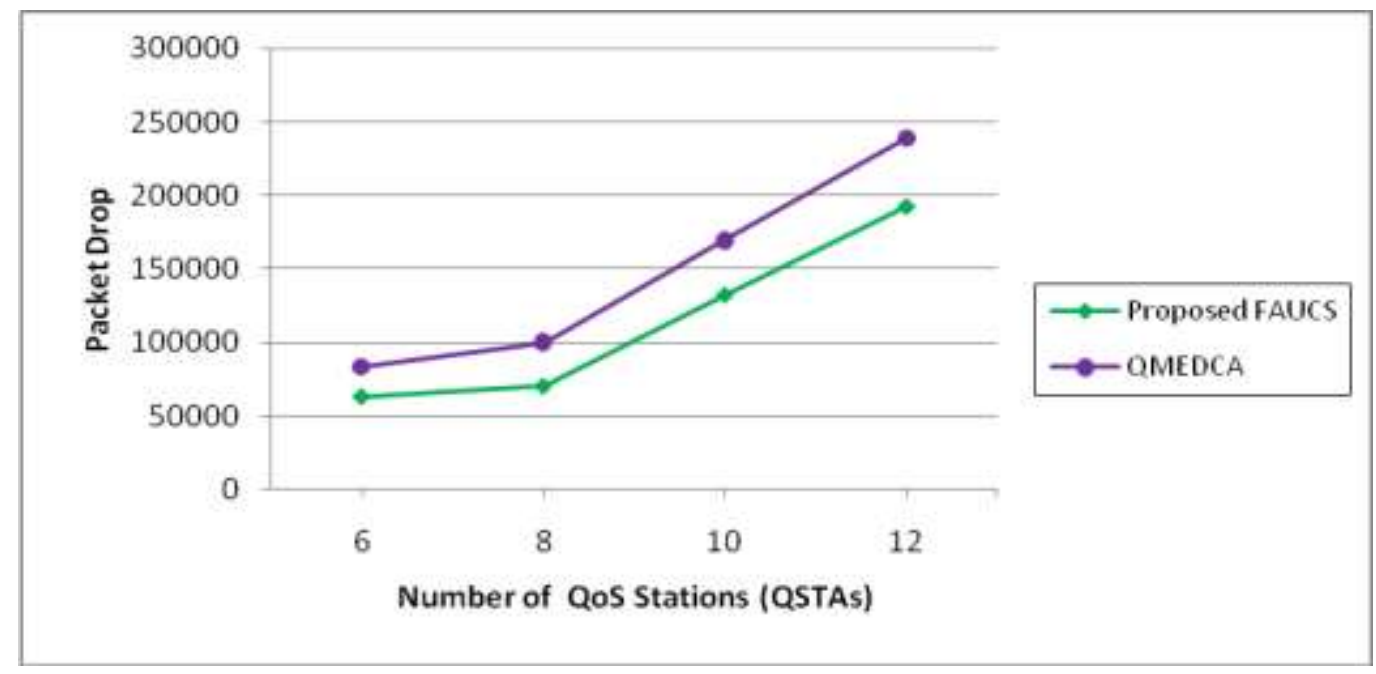

Figure 8. QoS Stations Vs Packet Drop 
The graph in Figure 8 shows the results of packet drop occurred for FAUCS and QMEDCA techniques when the number of QSTAs is varied. When increasing QSTAs, packet drop is also increases. As seen from the figure, the packet drop of FAUCS is increased from 62668 to 192168 and the packet drop of QMEDCA is increases from 83100 to 238821 . Hence, FAUCS has $24 \%$ lesser packet drop than QMEDCA technique.

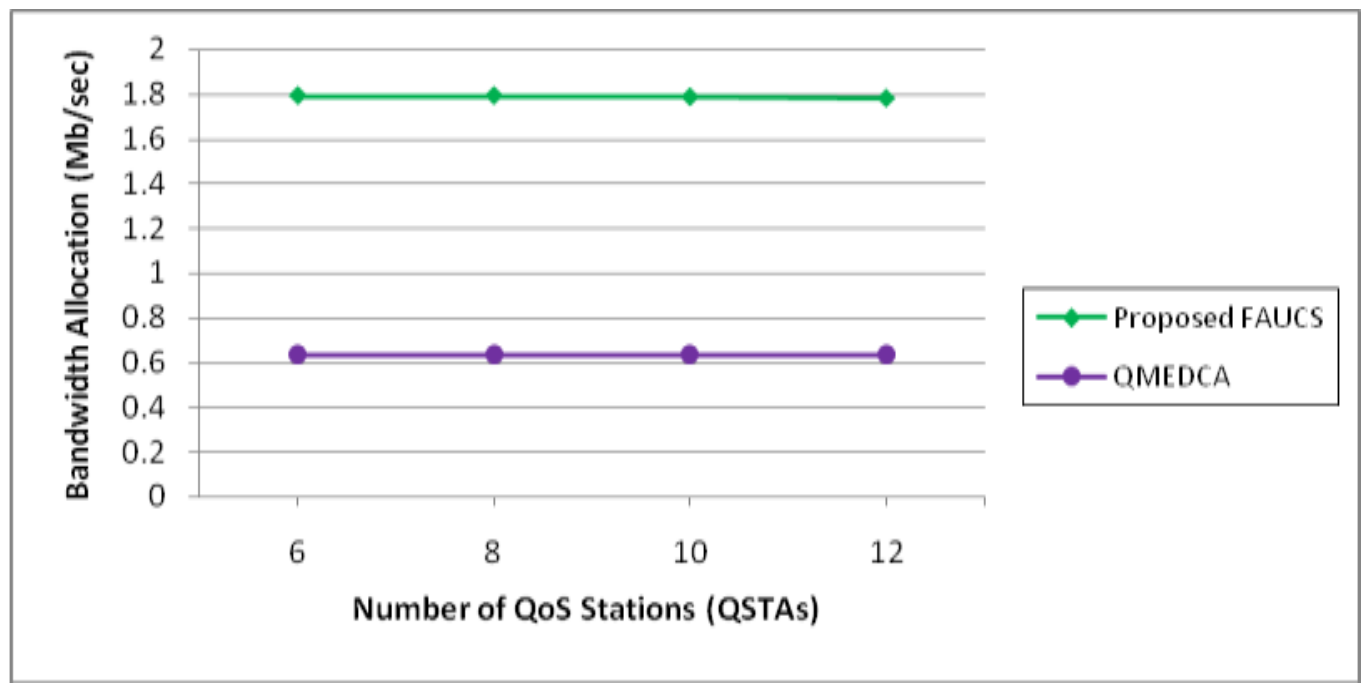

Figure 9. QoS Stations Vs Bandwidth Allocation

The graph in Figure 9 shows the results of bandwidth allocated occurred for FAUCS and QMEDCA techniques when the number of QSTAs is varied. When increasing QSTAs, allocated bandwidth remains almost constant. As seen from the figure, the allocated bandwidth of FAUCS remains constant around $1.78 \mathrm{Mb} / \mathrm{s}$ and the allocated bandwidth of QMEDCA remains constant around $0.63 \mathrm{Mb} / \mathrm{s}$. FAUCS has $64 \%$ higher bandwidth allocated than QMEDCA technique, it considers traffic AC also while estimating inter-node priority.

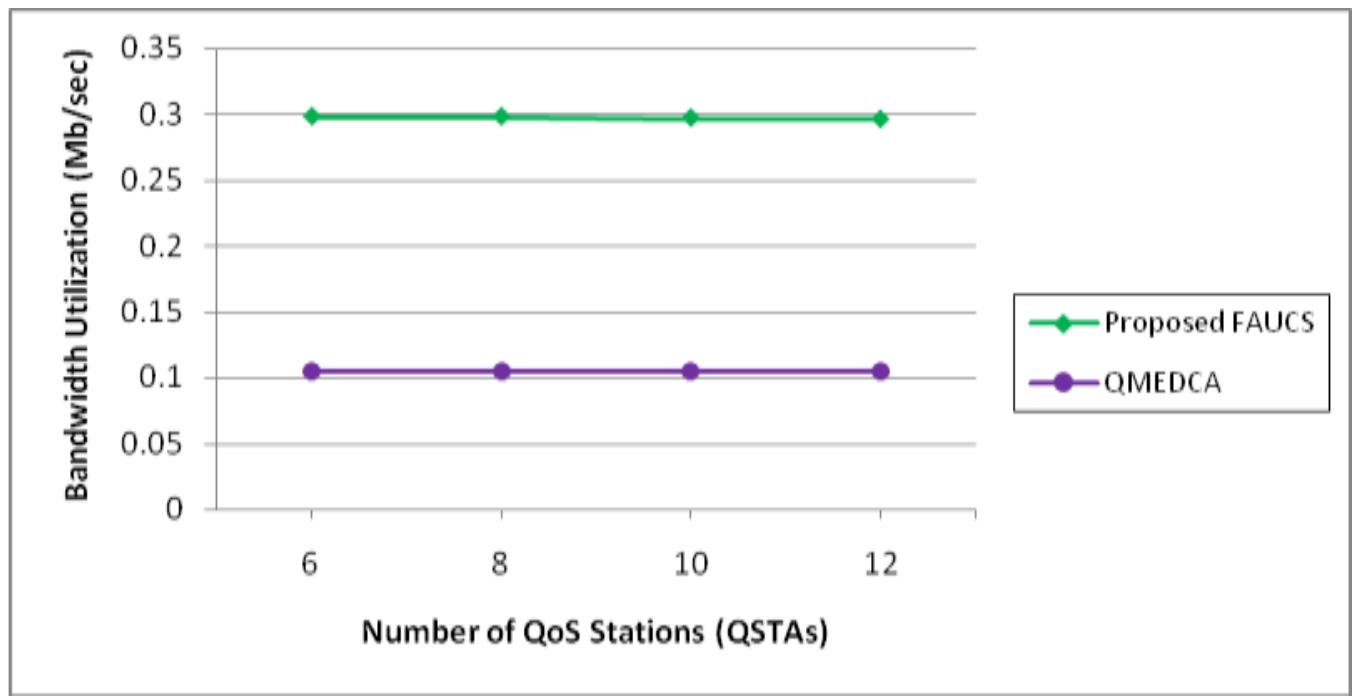

Figure 10. QoS Stations Vs Bandwidth Utilization

The graph in Figure 10 shows the results of bandwidth utilization occurred for FAUCS and QMEDCA techniques when the number of QSTAs is varied. When increasing QSTAs, bandwidth utilization remains almost constant. As seen from the figure, the 
bandwidth utilization of FAUCS remains constant around $0.29 \mathrm{Mb} / \mathrm{s}$ and the bandwidth utilization of QMEDCA remains constant around $0.10 \mathrm{Mb} / \mathrm{s}$. FAUCS has 65\% higher bandwidth utilization than QMEDCA technique, since it considers traffic AC also while estimating inter-node priority.

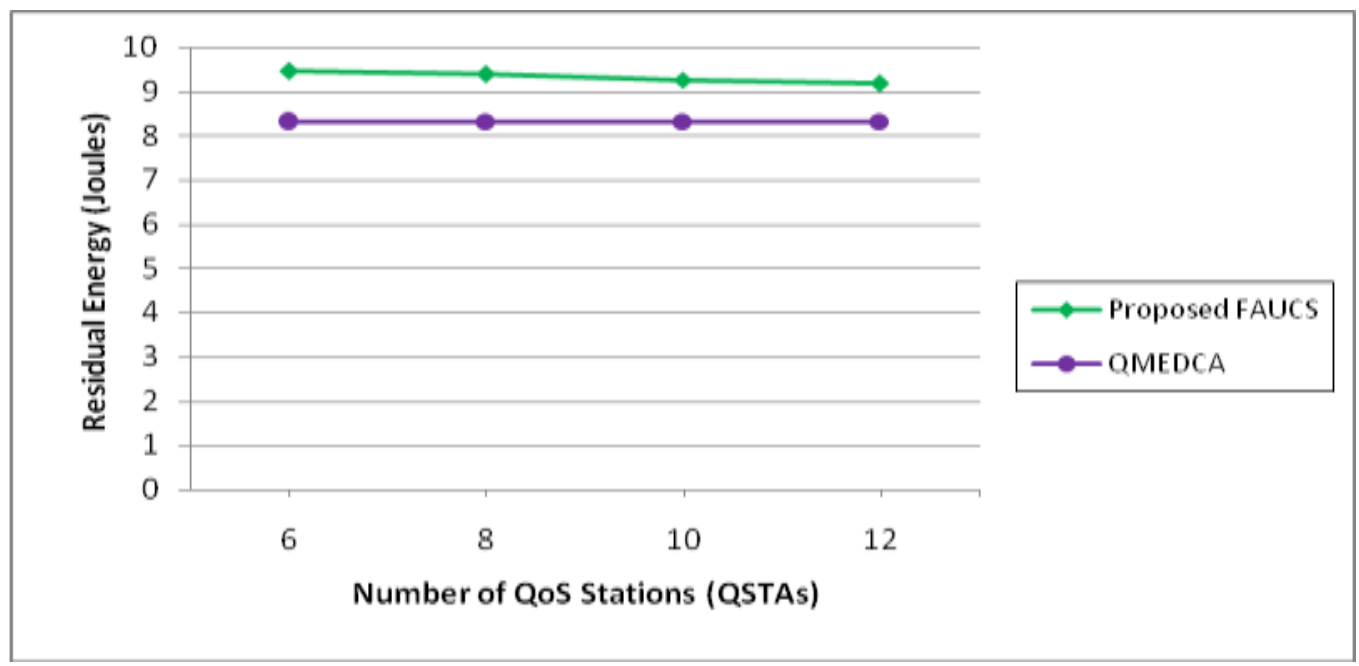

Figure 11. QoS Stations Vs Residual Energy

The graph in Figure 11 shows the results of residual energy occurred for FAUCS and QMEDCA techniques when the number of QSTAs is varied. When increasing QSTAs, residual energy decreases for FAUCS. As seen from the figure, the residual energy of FAUCS is decreased from 9.45 to 9.17 . Hence, FAUCS has $11 \%$ higher residual energy than QMEDCA technique.

4.3.2. Based on Transmission Rate: In the second experiment, the transmission rate is varies as $250 \mathrm{~kb}, 500 \mathrm{~kb}, 750 \mathrm{~kb}, 1000 \mathrm{~kb}, 1250 \mathrm{~kb}$ and $1500 \mathrm{~kb}$.

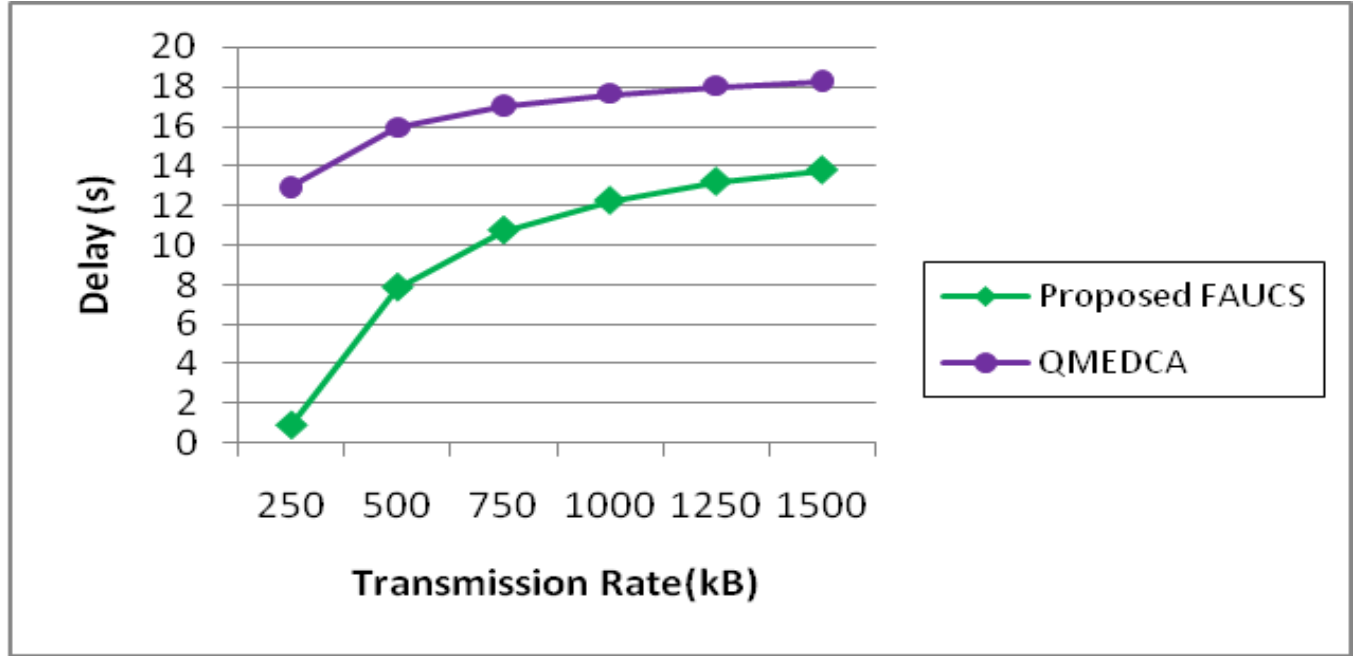

Figure 12. Transmission Rate Vs Delay

The graph in Figure 12 shows the results of delay occurred for FAUCS and QMEDCA techniques when the transmission rate is varied. When increasing transmission rate, delay is also increases. As seen from the figure, the delay of FAUCS is increased from 0.9 to 13.78 and the delay of QMEDCA is increases from 12.93 to 18.28. Hence, FAUCS has $44 \%$ lesser delay than QMEDCA technique. 


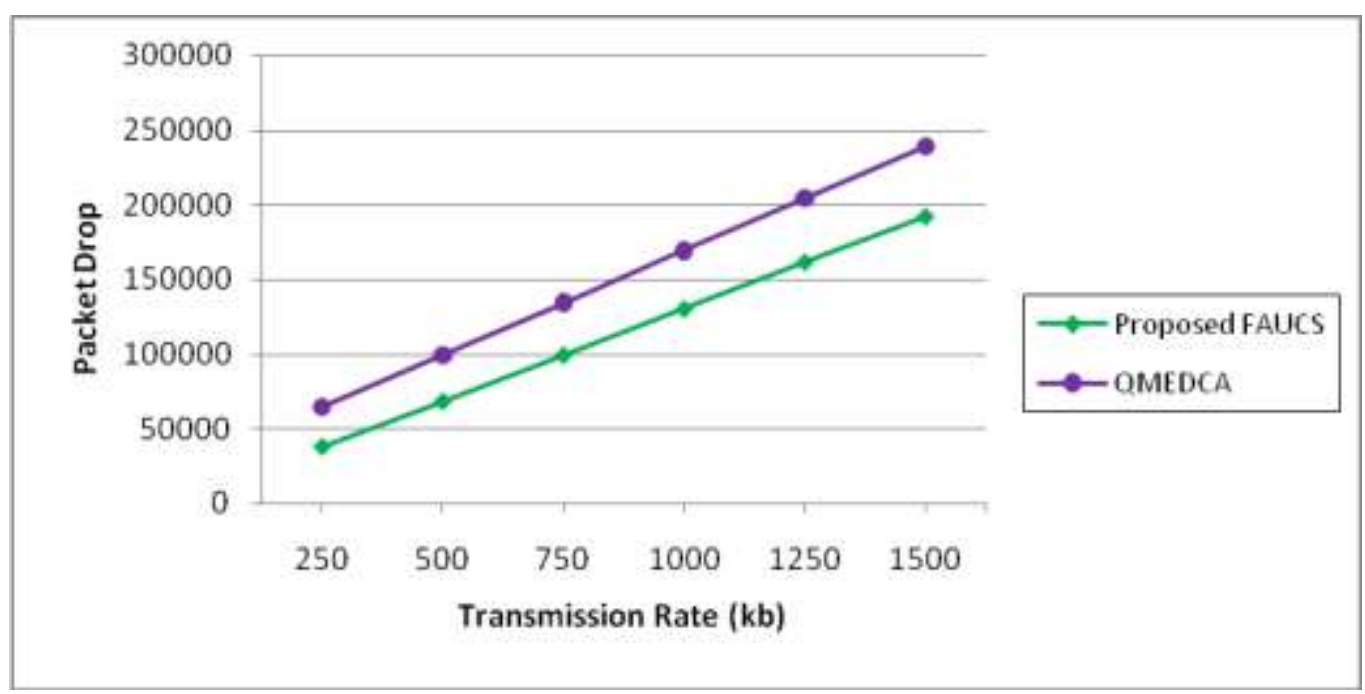

Figure 13. Transmission Rate Vs Packet Drop

The graph in Figure 13 shows the results of packet drop occurred for FAUCS and QMEDCA techniques when the transmission rate is varied. When increasing transmission rate, packet drop is also increases. As seen from the figure, the packet drop of FAUCS is increased from 37585 to 192168 and the packet drop of QMEDCA is increases from 64694 to 238821 . Hence, FAUCS has 27\% lesser packet drop than QMEDCA technique.

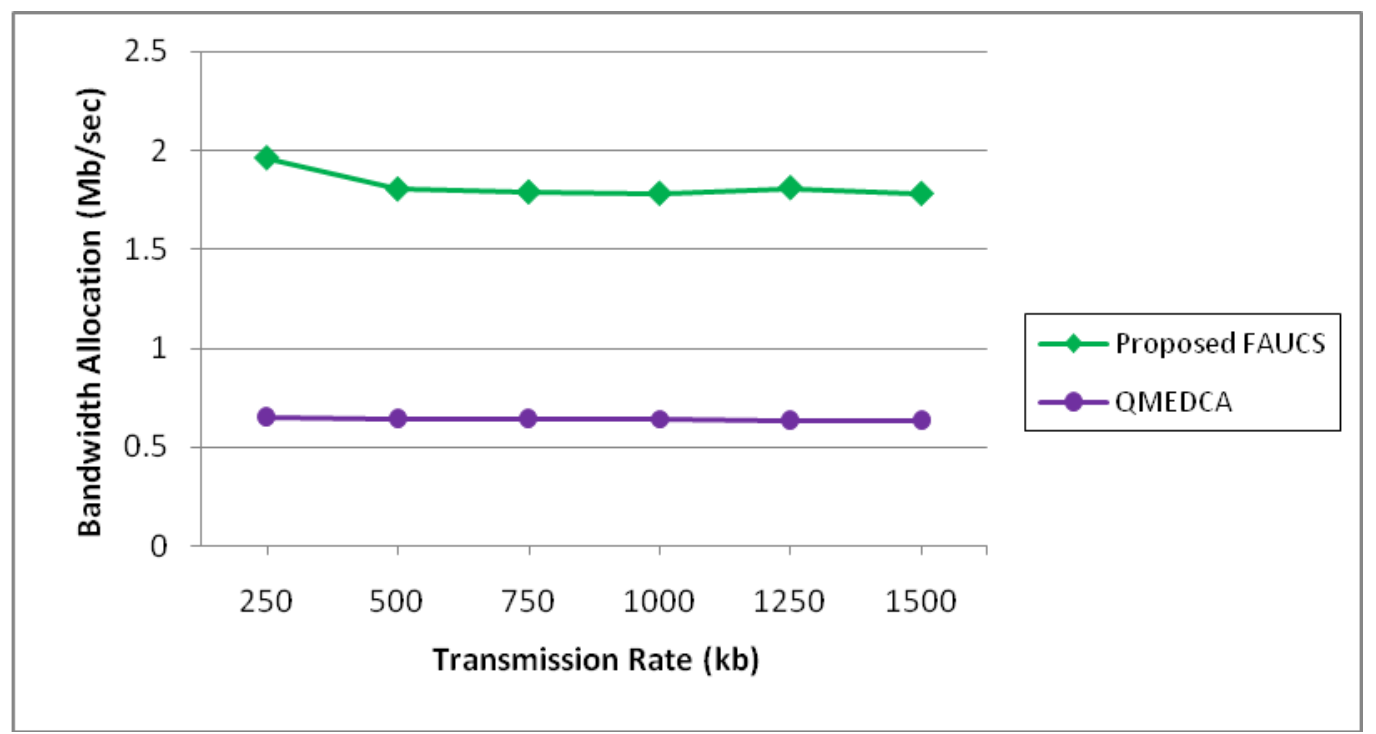

Figure 14. Transmission Rate Vs Bandwidth Allocation

The graph in Figure 14 shows the results of bandwidth allocated occurred for FAUCS and QMEDCA techniques when the transmission rate is varied. When increasing transmission rate, bandwidth is decreases. As seen from the figure, the bandwidth of FAUCS is decreased from 1.96 to 1.78 and the bandwidth of QMEDCA is decreases from 0.65 to 0.63 . Hence, FAUCS has $65 \%$ higher bandwidth allocated than QMEDCA technique. 


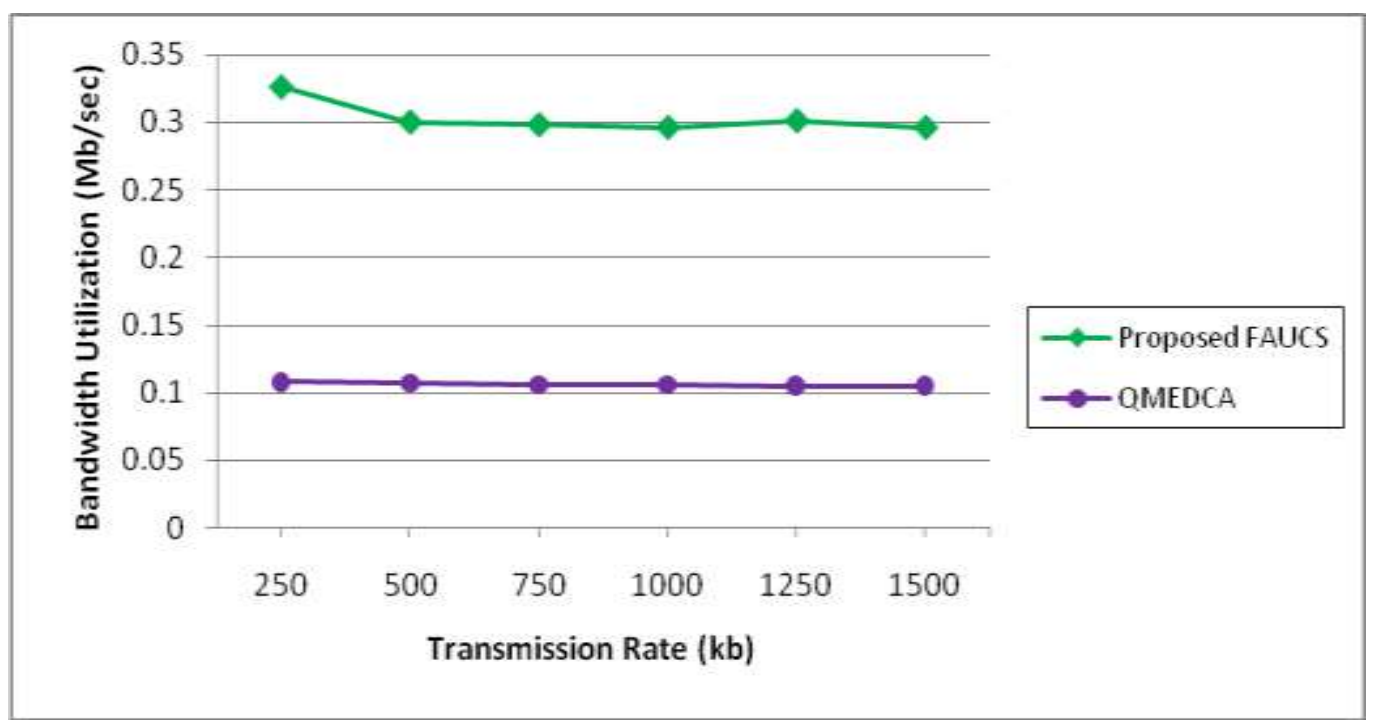

Figure 15. Transmission Rate Vs Bandwidth Utilization

The graph in Figure 15 shows the results of bandwidth utilization occurred for FAUCS and QMEDCA techniques when the transmission rate is varied. When increasing transmission rate, bandwidth is decreases. As seen from the figure, the bandwidth of FAUCS is decreased from 0.32 to 0.29 and the bandwidth of QMEDCA is decreases from 0.1088 to 0.1055 . Hence, FAUCS has $65 \%$ higher bandwidth utilization than QMEDCA technique.

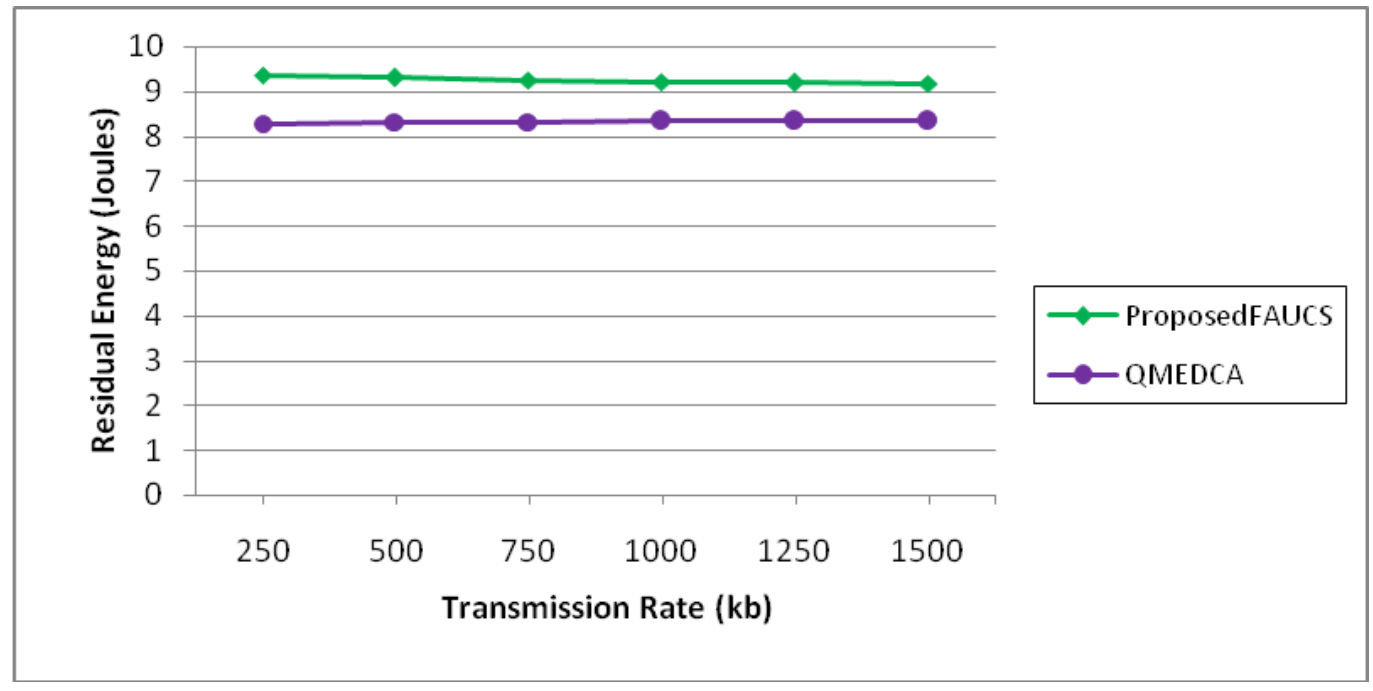

Figure 16. Transmission Rate Vs Residual Energy

The graph in Figure 16 shows the results of residual energy occurred for FAUCS and QMEDCA techniques when the transmission rate is varied. When increasing transmission rate, residual energy decreases for FAUCS. As seen from the figure, the residual energy of FAUCS is decreased from 9.36 to 9.17 . Hence, FAUCS has $10 \%$ higher residual energy than QMEDCA technique. 


\section{Conclusion}

In this paper, a Fuzzy based adaptive user-weight classification scheme (FAUCS) for EDCA in IEEE 802.11e WLAN is proposed. In this scheme, the inter-node priority for each traffic class is assigned to each mobile station. Fuzzy logic is applied to determine inter-node priority by considering the traffic access category, number of transmitted packets, collision rate and residual energy for each traffic class. Based on the outcome of the Fuzzy rules, inter node priority is determined and the node weights are updated dynamically. The proposed FAUCS is compared with the QM-EDCA and the performance is evaluated in terms of delay, packet drop, bandwidth allocation, bandwidth utilization and residual energy. Unlike the existing QM-EDCA system, the proposed FAUCS system provides user classification, has reduced delay and packet drop along with improved bandwidth utilization and residual energy.

\section{References}

[1] A. Politis, I. Mavridis and A. Manitsaris, "Enhancing Multimedia Traffic Performance in IEEE 802.11e Networks", IEEE Sixth International Conference on Wireless and Mobile Communications, Valencia, (2010).

[2] A. Poonguzhali, "Performance Evaluation of IEEE 802.11e MAC Layer using Cell Processor", International Journal of Scientific \& Technology Research, vol. 3, no. 1, (2014).

[3] H. J. Ji, C. C. Yu and F. H. Wen, "Flexible TXOP Assignments for Efficient QoS Scheduling in IEEE 802.11e WLANs", Fifth IFIP International Conference on Wireless and Optical Communications Networks, (2008).

[4] M. Ricardo, P. Paulo, V. Francisco and F. Ricardo, "Assessment of the IEEE 802.11e EDCA Protocol Limitations when Dealing with Real-Time Communication", EURASIP Journal on Wireless Communications and Networking, (2010), pp. 1-14.

[5] H. A. Ngo and T. G. Pham, "An Enhanced MAC-Layer Improving to Support QoS for Multimedia Data in Wireless Networks", Indian Journal of Science and Technology, vol. 9, no. 20, (2016), pp. 1-7.

[6] T. Hicham and F. Youssef, "A Fuzzy-based QoS Maximization Protocol for WiFi Multimedia (IEEE 802.11e) Ad hoc Networks", International Journal of Communication Networks and Information Security, vol. 6, no. 3, (2014), pp. 217-225.

[7] Q. Hua and Y. Kenji, "Adaptive QoS Admission Control for IEEE 802.11e Network", Fifth IEEE Consumer Communications and Networking Conference, Las Vegas, (2008).

[8] R. R. Ahmed and H. Saïd, "A Dynamic Multimedia User-Weight Classification Scheme For IEEE 802.11 WLANs", International Journal of Computer Networks and Communications, vol. 3, no. 2, (2011).

[9] D. Pingping, W. Jianxin, W. Haodong and P. Yi, "Boosting VoIP Capacity via Service Differentiation in IEEE 802.11e EDCA Networks", International Journal of Distributed Sensor Networks, vol. 2015, (2015), pp.1-11.

[10] K. Sunmyeng, "Cooperative MAC Protocol based on TXOP and Block ACK in IEEE 802.11e WLANs", International Journal of Engineering and Technology, Engineering Journals Publications, vol. 6, no. 5, (2014), pp. 2440-2451.

[11] S. Pablo, B. Albert, P. Paul Patras and A. Arturo, "Optimal Configuration of 802.11e EDCA for RealTime and Data Traffic", IEEE Transactions on Vehicular Technology, vol. 59, no. 5, (2010), pp. 25112527.

[12] Y. L. Kang, S. C. Kee and R. Won, "Efficient QoS Scheduling Algorithm for Multimedia Services in IEEE 802.11e WLAN", IEEE Vehicular Technology Conference, (2011).

[13] M. Saeid, F. Mahmood and B. Reza, "An Adaptive Fair-Distributed Scheduling Algorithm to Guarantee QoS for Both VBR and CBR Video Traffics on IEEE 802.11e WLANs", EURASIP Journal on Advances in Signal Processing, (2008).

[14] U. A. Fakhar and K. S. Shikhar, "QoS and Admission Controller in IEEE 802.11e WLAN", Fourth International Conference on Intelligent Systems, Modelling and Simulation, (2013).

[15] K. Feyza, I. Inanc and A. Ender, "Weighted Fair Uplink/Downlink Access Provisioning in IEEE 802.11e WLANs", IEEE International Conference on Communications, Beijing, (2008). 


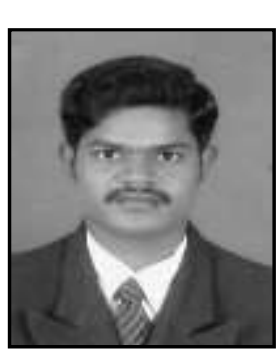

\section{Authors}

V. Ilayaraja, was born in Tamilnadu, India, in the year 1984. He obtained his Bachelor's degree in Information Technology from Anna University, Chennai in the year 2005. Then, he obtained his Master's degree in Computer Science and Engineering from Anna University, Chennai in the year 2007. Currently, he is an Assistant Professor (Sr.Gr) at the Department of Information Technology, PSG College of Technology, Coimbatore, Tamilnadu, India. His Specializations include Wireless Networks, Wireless LAN, Mobile Computing and Database Management Systems. His current research interest is an enhancement of QoS in Wireless LAN.

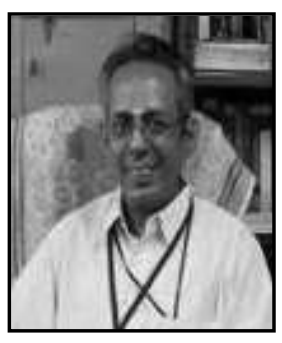

R. Venkatesan, was born in Tamilnadu, India, in the year 1958. He received his B.E (Hons) degree from Madras University in the year 1980. He completed his Masters degree in Industrial Engineering from Madras University in the year 1982. He obtained his second Masters degree MS in Computer and Information Science from University of Michigan, USA in the year 1999. He was awarded with PhD from Anna University, Chennai in the year 2007. He is currently Professor and Head in the Department of Computer Science and Engineering at PSG College of Technology, Coimbatore, Tamilnadu, India. His research interests are in Simulation and Modeling, Software Engineering, Algorithm Design and Software Process Management. 
International Journal of Future Generation Communication and Networking Vol. 10, No. 1 (2017) 